\title{
A practical probabilistic evaluation method for the noise reduction effect of sound absorbing materials
}

\author{
Yasuo Mitani and Mitsuo Ohta \\ Faculty of Engineering, Hiroshima University, \\ Shitami, Saijo-cho, Higashi-Hiroshima, 724 Japan
}

(Received 18 May 1987)

\begin{abstract}
This report describes a practical probabilistic evaluation method for the stochastic noise reduction effect when a stationary random noise excitation of arbitrary non-Gaussian distribution type is attenuated by inserting a sound absorbing material into a room. The effectiveness of the proposed method has been confirmed by applying it to the actual noise data observed in a reverberation room. The experimental results are in good agreement with the theoretically predicted curves.
\end{abstract}

PACS number: 43. 55. Ev, 43. 60. $\mathrm{Cg}$

\section{INTRODUCTION}

In the practical engineering field of noise control, a sound absorbing material is very often inserted into a room, to produce the attenuation of acoustic noise. The acoustical design and/or the evaluation problem of sound absorbing materials have been already considered by many investigators. ${ }^{1-3)}$ Almost all of these studies, however, were confined only to the effects based on the deterministic signals or the gross average evaluation of noise reduction due to the sound absorbing materials.

In the practical problem of the acoustical design and/or the evaluation of sound absorbing material, the following points have to be taken into consideration:

(i) The living environmental noises which one encounters in daily life exhibit various types of probability distributions, apart from a standard Gaussian distribution, due to the diversified causes of fluctuation.

(ii) As is well-known, every information on the statistical evaluation indices such as $L_{\mathrm{eq}}$, median, $L_{x}(x=5,10, \ldots$; i.e., $(100-x)$ percentage point of the level distribution) can be evaluated by first finding the probability distribution function of random noise. (iii) A usual noise evaluation index $L_{x}$ must be closely connected with the statistical properties (e.g., the probability distribution characteristics, the power frequency spectrum and its temporal change, etc.) of the input noise fluctuation emitted from sound sources and the frequency characteristics of the sound absorbing material.

In this report, a practical probabilistic evaluation method for predicting the noise level distribution is derived for the actual cases with an incident random noise of an arbitrary probability distribution type to the sound absorbing material. The main concern in this study is focussed on how to predict the noise evaluation index $L_{x}$ by use of the information on the statistical properties of random noise and the frequency characteristic of the sound absorbing material. Finally, the effectiveness of the present method is experimentally confirmed by applying it to the actual noise attenuation data observed in a reverberation room.

\section{THEORETICAL CONSIDERATION}

2.1 General Theory on Noise Level Distribution

For the purpose of predicting the noise evaluation index, $L_{x}$, it is first necessary to find generally an explicit expression for the noise level probability distribution function. In order to obtain the precise 
evaluation of complicated random phenomena like environmental noises, it would be necessary to employ a general probability expression of a series expansion type with many parameters. Thus, let us introduce the statistical Hermite series expansion type expression as a general explicit expression on the probability density function, $P(x)$, with respect to the noise level fluctuation $x$, as follows ${ }^{4}$ :

$$
\begin{aligned}
P(x)= & N\left(x ; \mu_{x}, \sigma_{x}^{2}\right) \\
& +\sum_{n=3}^{\infty} A_{n} H_{n}\left(\frac{x-\mu_{x}}{\sigma_{x}}\right) N\left(x ; \mu_{x}, \sigma_{x}^{2}\right),
\end{aligned}
$$

with

$$
\begin{aligned}
& \mu_{x}=\langle x\rangle, \quad \sigma_{x}^{2}=\left\langle\left(x-\mu_{x}\right)^{2}\right\rangle, \\
& A_{n} \triangleq \frac{1}{n !}\left\langle H_{n}\left(\frac{x-\mu_{x}}{\sigma_{x}}\right)\right\rangle,
\end{aligned}
$$

where $\langle *\rangle$ denotes an averaging operation with respect to the random variable $*$ and $N\left(x ; \mu_{x}, \sigma_{x}^{2}\right)$ denotes the Gaussian distribution function:

$$
N\left(x ; \mu_{x}, \sigma_{x}^{2}\right) \triangleq \frac{1}{\sqrt{2 \pi} \sigma_{x}} \exp \left[-\left(x-\mu_{x}\right)^{2} / 2 \sigma_{x}^{2}\right] .
$$

At this time, by using the well-known formula between the Gaussian distribution function and the Hermite polynomial ${ }^{4)}$ :

$$
\begin{aligned}
& N\left(x ; \mu_{x}, \sigma_{x}^{2}\right) H_{n}\left(\frac{x-\mu_{x}}{\sigma_{x}}\right) \\
& \quad=(-1)^{n} \sigma_{x}{ }^{n} \frac{d^{n}}{d x^{n}} N\left(x ; \mu_{x}, \sigma_{x}^{2}\right),
\end{aligned}
$$

the cumulative distribution form $Q(x)\left(\triangleq \int_{-\infty}^{x} P(\xi) d \xi\right)$ is consequently obtained as follows:

$$
\begin{aligned}
Q(x)= & \int_{-\infty}^{x} N\left(\xi ; \mu_{x}, \sigma_{x}^{2}\right) d \xi \\
& -\sigma_{x} \cdot N\left(x ; \mu_{x}, \sigma_{x}^{2}\right) \sum_{n=3}^{\infty} A_{n} H_{n-1}\left(\frac{x-\mu_{x}}{\sigma_{x}}\right) .
\end{aligned}
$$

In this expression, the statistical information on lower and higher order moments for an incident noise fluctuation closely connected with the frequency characteristic of a sound absorbing material is reflected explicitly in each expansion coefficient $A_{n}$. The objective $L_{x}$ noise evaluation indices (e.g., $\left.L_{5}, L_{10}, L_{50}, L_{90}, \ldots\right)$ can be directly evaluated by this cumulative distribution form. Furthermore, it should be noticed that Eq. (5) (or Eq. (1)) is an orthogonal expansion series type expression which shows reasonably the hierachical property without the redundancy of statistical information on the random phenomenon under consideration.

2.2 Relationship between the Output Distribution Parameters and the Frequency Characteristics for a Sound Absorbing Material

As is well-known, the noise level fluctuation $x$ in a $\mathrm{dB}$ scale is defined as:

$$
x=10 \log _{10} \frac{e}{e_{0}},
$$

where $e_{0}$ is a reference noise intensity usually taken as $10^{-12} \mathrm{~W} / \mathrm{m}^{2}$ and $e$ is the noise intensity fluctuation under consideration. Thus, in order to predict the objective cumulative distribution form $Q(x)$ for the output noise level fluctuation $x$ after inserting a sound absorbing material into a room, first one has to evaluate the output noise intensity fluctuation, $e$, in relation to the frequency characteristics of the sound absorbing material.

Let $Y_{i}(i=1,2, \ldots, N)$ be the input noise intensity fluctuation existing in the $i$ th frequency band before inserting a sound absorbing material and $E_{i}$ ( $i=$ $1,2, \ldots, N)$ be the output noise intensity fluctuation after inserting the sound absorbing material. Moreover, let the noise attenuation coefficient, $a_{i}(i=$ $1,2, \ldots, N)$, denote the power frequency characteristic of the sound absorbing material at a center frequency, $f_{\text {ci }}$, of the $i$ th one-third-octave band (or octave band). Based on the additive property of energy quantities, therefore, the over-all noise intensity fluctuation after inserting the sound absorbing material is directly given by:

$$
e=\sum_{i=1}^{N} a_{i} Y_{i} .
$$

Originally, the above power frequency characteristic, $a_{i}(i=1,2, \ldots, N)$, can be simply estimated by use of the above two data $Y_{i}$ and $E_{i}$, as follows:

$$
a_{i}=\frac{\left\langle E_{i}\right\rangle}{\left\langle Y_{i}\right\rangle} .
$$

Of course, it must be noticed that this parameter $a_{i}$ can get a constant value in every actual cases with an arbitrary random input, though it is estimated by use of the only two lower order moments as shown in Eq. (8) as a special case. Accordingly, the $p$ th order moment, $\left\langle e^{p}\right\rangle(p=1,2, \ldots)$, for $e$ can be estimated as:

$$
\left\langle e^{p}\right\rangle=\sum_{i+j+\cdots+n=p} \frac{p !}{i ! j ! \cdots n !} a_{1}^{i} a_{2}^{j} \cdots a_{N}^{n}\left\langle Y_{1}^{i} \cdot Y_{2}^{j} \cdots Y_{N}^{n}\right\rangle,
$$




\section{Y. MITANI and M. OHTA: PROBABILISTIC EVALUATION OF SOUND ABSORBING MATERIALS}

by use of the statistical information on the frequency component, $Y_{i}(i=1,2, \ldots, N)$, of the incident noise intensity fluctuation and the estimated power frequency characteristic, $a_{i}(i=1,2, \ldots, N)$, of the sound absorbing material.

On the other hand, for the purpose of finding the fundamental relationship between the moment statistics, $\left\langle e^{p}\right\rangle(p=1,2, \ldots)$, for the noise intensity fluctuation $e$ and the cumulant statistics, $\kappa_{x, p}$ ( $p=$ $1,2, \ldots)$, for the noise level fluctuation $x$, the moment generating function, $m_{x}(\theta)$, for $x$ is considered as the starting point of the analysis, as follows:

$$
\begin{aligned}
m_{x}(\theta) & \triangleq\left\langle e^{\theta x}\right\rangle \\
& =\left\langle\exp \left[\theta c \ln \frac{e}{e_{0}}\right]\right\rangle \\
& =\left\langle\left(\frac{e}{e_{0}}\right)^{\theta c}\right\rangle
\end{aligned}
$$

with

$$
c \triangleq \frac{10}{\ln 10}
$$

At this time, by replacing the above parameter $\theta c$ with $m$ in Eq. (10), one can easily obtain the following expression:

$$
m_{x}\left(\frac{m}{c}\right)=\left\langle\left(\frac{e}{e_{0}}\right)^{m}\right\rangle=\frac{1}{e_{0}{ }^{m}}\left\langle e^{m}\right\rangle .
$$

Moreover, from the mathematical viewpoint, $m_{x}(m / c)$ can be also expressed $\mathrm{as}^{5)}$ :

$$
m_{x}\left(\frac{m}{c}\right)=\exp \left[\sum_{p=1}^{\infty} \frac{\kappa_{x, p}}{p !}\left(\frac{m}{c}\right)^{p}\right],
$$

where $\kappa_{x, p}$ denotes the $p$ th order cumulant for $x$. Thus, from Eqs. (11) and (12), the fundamental relationship between $\kappa_{x, p}$ and $\left\langle e^{m}\right\rangle$ can be consequently obtained as:

$$
\sum_{p=1}^{\infty} \frac{\kappa_{x, p}}{p !}\left(\frac{m}{c}\right)^{p}=\ln \frac{\left\langle e^{m}\right\rangle}{e_{0}{ }^{m}} .
$$

By solving the simultaneous equations derived from the above equation (for the $p$ th order cumulant $\kappa_{x, p}$ $(p=1,2, \ldots, M))$ :

$$
\left[\begin{array}{ccccc}
\frac{1}{c} & \frac{1}{2 !}\left(\frac{1}{c}\right)^{2} & \frac{1}{3 !}\left(\frac{1}{c}\right)^{3} & \cdots \cdots & \frac{1}{M !}\left(\frac{1}{c}\right)^{M} \\
\frac{2}{c} & \frac{1}{2 !}\left(\frac{2}{c}\right)^{2} & \frac{1}{3 !}\left(\frac{2}{c}\right)^{3} & \cdots \cdots & \frac{1}{M !}\left(\frac{2}{c}\right)^{M} \\
\frac{3}{c} & \frac{1}{2 !}\left(\frac{3}{c}\right)^{2} & \frac{1}{3 !}\left(\frac{3}{c}\right)^{3} & \cdots \cdots & \frac{1}{M !}\left(\frac{3}{c}\right)^{M} \\
\vdots & \vdots & \vdots & \ddots & \vdots \\
\vdots & \vdots \\
\frac{M}{c} & \frac{1}{2 !}\left(\frac{M}{c}\right)^{2} & \frac{1}{3 !}\left(\frac{M}{c}\right)^{3} & \cdots \cdots & \frac{1}{M !}\left(\frac{M}{c}\right)^{M}
\end{array}\right]\left[\begin{array}{c}
\kappa_{x, 1} \\
\kappa_{x, 2} \\
\kappa_{x, 3} \\
\vdots \\
\kappa_{x, M}
\end{array}\right]=\left[\begin{array}{c}
\ln \frac{\langle e\rangle}{e_{0}} \\
\ln \frac{\left\langle e^{2}\right\rangle}{e_{0}^{2}} \\
\ln \frac{\left\langle e^{3}\right\rangle}{e_{0}^{3}} \\
\vdots \\
\ln \frac{\left\langle e^{M}\right\rangle}{e_{0}^{M}}
\end{array}\right],
$$

the objective cumulant statistics $\kappa_{x, p}$ can be given. Hereupon, in view of the following experimental consideration, the solution for $M=4$ is given by:

$$
\begin{aligned}
\kappa_{x, 1}= & c\left\{\ln \frac{1}{e_{0}}+4 \ln \langle e\rangle-3 \ln \left\langle e^{2}\right\rangle\right. \\
& \left.+\frac{4}{3} \ln \left\langle e^{3}\right\rangle-\frac{1}{4} \ln \left\langle e^{4}\right\rangle\right\}, \\
\kappa_{x, 2}= & c^{2}\left\{-\frac{26}{3} \ln \langle e\rangle+\frac{19}{2} \ln \left\langle e^{2}\right\rangle\right. \\
& \left.-\frac{14}{3} \ln \left\langle e^{3}\right\rangle+\frac{11}{12} \ln \left\langle e^{4}\right\rangle\right\}, \\
\kappa_{x, 3}= & c^{3}\left\{9 \ln \langle e\rangle-12 \ln \left\langle e^{2}\right\rangle+7 \ln \left\langle e^{3}\right\rangle\right. \\
& \left.-\frac{3}{2} \ln \left\langle e^{4}\right\rangle\right\},
\end{aligned}
$$

$$
\begin{aligned}
\kappa_{x, 4}= & c^{4}\left\{-4 \ln \langle e\rangle+6 \ln \left\langle e^{2}\right\rangle-4 \ln \left\langle e^{3}\right\rangle\right. \\
& \left.+\ln \left\langle e^{4}\right\rangle\right\} .
\end{aligned}
$$

Thus, the output distribution parameters $\mu_{x}, \sigma_{x}^{2}$ and $A_{n}$ in Eq. (5) can be calculated by use of the above $\kappa_{x, p}$ and the statistical relationship ${ }^{6)}$ :

$$
\left.\begin{array}{l}
\langle x\rangle=\kappa_{x, 1}, \\
\left\langle x^{2}\right\rangle=\kappa_{x, 2}+\kappa_{x, 1}^{2}, \\
\left\langle x^{3}\right\rangle=\kappa_{x, 3}+3 \kappa_{x, 1} \cdot \kappa_{x, 2}+\kappa_{x, 1}^{3}, \\
\left\langle x^{4}\right\rangle=\kappa_{x, 4}+3 \kappa_{x, 2}^{2}+4 \kappa_{x, 1} \cdot \kappa_{x, 3}+6 \kappa_{x, 1}^{2} \cdot \kappa_{x, 2}+\kappa_{x, 1}^{4} .
\end{array}\right\}
$$

Substituting the obtained distribution parameters $\mu_{x}, \sigma_{x}{ }^{2}$ and $A_{n}$ into Eq. (5), one can concretely obtain the objective cumulative noise level distribution. 


\section{EXPERIMENTAL CONSIDERATION}

The experiment was done in a reverberation room and the block diagram of the experimental arrangement is shown in Fig. 1. Using a band-pass filter and an amplifier, a white noise with the band width from $224 \mathrm{~Hz}$ to $447 \mathrm{~Hz}$, generated from a random noise generator, was supplied to a loudspeaker. The received acoustic noise wave observed at the specific receiving point (see Fig. 1) was recorded by use of a data recorder. The observed values of the averaged noise intensities existing in the $i$ th frequency band before and after inserting a sound absorbing material made of porous material (35.5 (width) $\times 15.5$ (height) $\times 69.0$ (length) $\mathrm{cm}$ ) are shown in Table 1 . Hereupon, the one-third-octave band analysis was used. The frequency characteristic, $a_{i}$, of the sound absorbing material is directly estimated by use of Eq. (8) and shown in Table 2.

For confirming the effectiveness of only the probability expression, Eq. (5), a comparison between the theoretically evaluated probability distribution curves by using the moment statistics of the output noise level fluctuation $x$ observed after inserting the sound absorbing material and the experimentally sampled

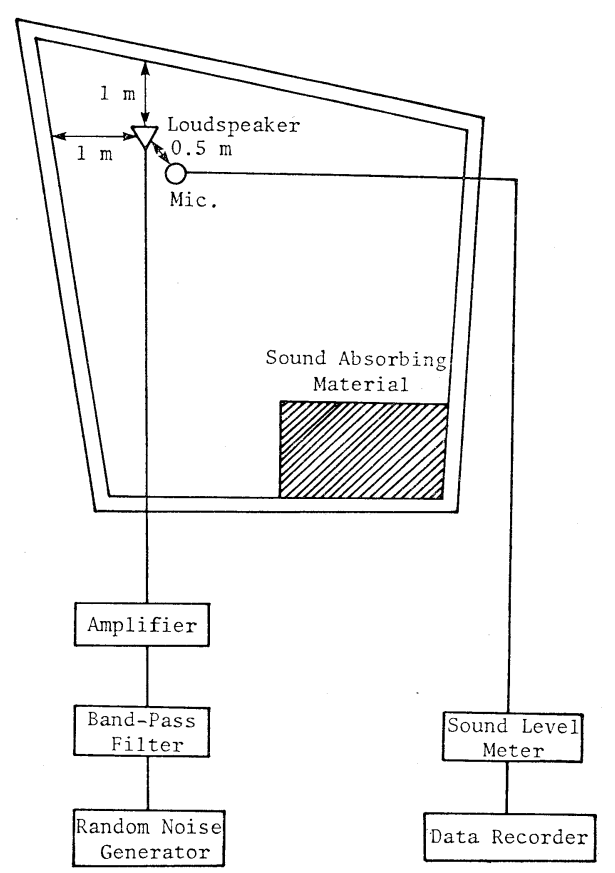

Fig. 1 The block diagram of experimental arrangement.
Table 1 The averaged noise intensities before and after inserting the sound absorbing material into the reverberation room.

\begin{tabular}{ccc}
\hline $\begin{array}{c}1 / 3 \text { Octave } \\
\text { band center } \\
\text { frequency } \\
(\mathrm{Hz})\end{array}$ & $\begin{array}{c}\text { Averaged noise } \\
\text { intensity before } \\
\text { inserting the sound } \\
\text { absorbing material } \\
\left(\mathrm{W} / \mathrm{m}^{2}\right)\end{array}$ & $\begin{array}{c}\text { Averaged noise } \\
\text { intensity after } \\
\text { inserting the sound } \\
\text { absorbing material } \\
\left(\mathrm{W} / \mathrm{m}^{2}\right)\end{array}$ \\
\hline 250 & $4.135 \times 10^{-3}$ & $2.528 \times 10^{-3}$ \\
315 & $4.851 \times 10^{-3}$ & $3.711 \times 10^{-3}$ \\
400 & $4.294 \times 10^{-3}$ & $2.860 \times 10^{-3}$ \\
\hline
\end{tabular}

Table 2 Power frequency characteristic $a_{i}$ of the sound absorbing material.

\begin{tabular}{cc}
\hline $\begin{array}{c}1 / 3 \\
\text { Octave band center } \\
\text { frequency }(\mathrm{Hz})\end{array}$ & $\begin{array}{c}\text { Power frequency } \\
\text { characteristic }\end{array}$ \\
\hline 250 & 0.6114 \\
315 & 0.7650 \\
400 & 0.6660 \\
\hline
\end{tabular}

points is shown in Fig. 2. Accordingly, the effect of the sound absorbing material is resultantly and experimentally reflected in each expansion coefficient $A_{n}$ of Eq. (5). Hereupon, let us define an expansion expression from the first term to the term with expansion coefficient $A_{n}(n \geq 3)$ in Eq. (5) as the (n2)th approximation of $Q(x)$. From this figure, the only first expansion term agrees approximately with the experimentally sampled points. By reviewing this figure in detail, however, it is obvious that the successive addition of higher expansion terms moves the theoretically estimated curves closer to the experimentally sampled points. In the following, let us evaluate the output probability distribution after inserting the sound absorbing material based on the incident noise intensity data, $Y_{i}(i=1,2,3)$, before inserting the sound absorbing material and the estimated power frequency characteristic, $a_{i}(i=1,2$, 3 ), for the sound absorbing material itself.

Figure 3 shows a comparison between the theoretically predicted curves by use of the proposed method and the experimentally sampled points in the form of a cumulative noise level probability distribution (after inserting the sound absorbing material), together with the actual data observed before inserting the sound absorbing material. From this figure, the prediction errors of the noise evaluation indices such as $L_{5}, L_{10}$ and $L_{50}$ usually used in the actual noise 


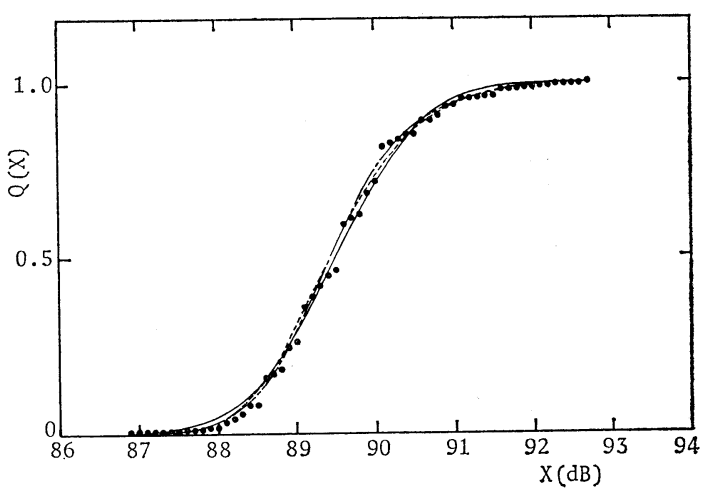

Fig. 2 A comparison between theoretically estimated curves by use of Eq. (5) and experimentally sampled points in the form of a cumulative noise level distribution (after inserting the sound absorbing material into the reverberation room). Experimentally sampled points are marked by and theoretically estimated curves by use of Eq. (5) are respectively shown as- $\longrightarrow$, the first term of Eq. (5); ----, the first approximation; - - - , the second approximation.

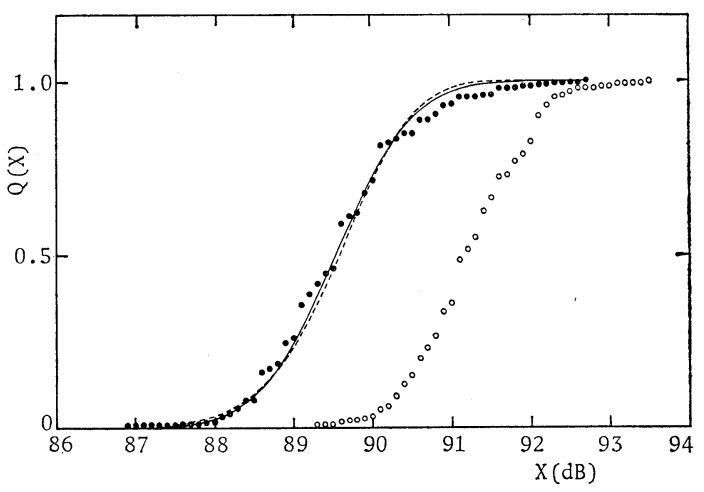

Fig. 3 A comparison between theoretically predicted curves by use of the proposed method and experimentally sampled points in the form of a cumulative noise level distribution (after inserting the sound absorbing material into the reverberation room). Experimentally sampled points are marked by and theoretically predicted curves by use of the proposed method are respectively shown as first term of Eq. (5); - ---, the first approximation. Experimentally sampled points before inserting the sound absorbing material are marked by $\bigcirc$. evaluation and/or regulation problems are almost all within $\pm 1 \mathrm{~dB}$.

It should be noticed that the proposed prediction method is able to treat any kind of random phenomena once if the power frequency characteristic is given experimentally or theoretically in advance.

\section{CONCLUSION}

A practical method for predicting the cumulative probability distribution for the noise level fluctuation in a room has been theoretically proposed, for a stationary random noise of an arbitrary distribution type attenuated by inserting the sound absorbing material into the room. Especially from the practical point of view, the power frequency characteristic of the sound absorbing material has been simply estimated from the actual noise data before and after inserting the sound absorbing material. The effect of this power frequency characteristic and the statistical properties of the input noise intensity fluctuation emitted from a sound source are reflected in each distribution parameter of the series expansion type probability expression. The effectiveness of the proposed theoretical evaluation method has been experimentally confirmed by applying it to the actual noise data.

Since the research on this kind of probabilistic evaluation of sound absorbing materials is in an early stage of study, main point of this report is focussed on introducing especially its principal viewpoint of practicality. Accordingly, there still remain many problems for the future studies, as follows:

1) This evaluation method should be applied to many other actual cases to broaden and confirm its practical effectiveness.

2) It is necessary to find the concrete structural relationship between the sound absorbing materials with various qualities of the materials and an incomplete diffuse sound field.

3) Based on the above relationship, it is also necessary to establish the systematical evaluation method for the objective probabilistic reduction effect by considering various physical mechanisms such as the spatial propagation characteristic in an incomplete diffuse sound field, the absorption coefficient of a sound absorbing material, its quality of the material, etc.

\section{ACKNOWLEDGEMENTS}

The authors would like to express their greateful 
thanks to Prof. S. Yamaguchi, Dr. K. Hatakeyama and Mr. U. Yoh for their helpful advice and assistance.

\section{REFERENCES}

1) C. M. Harris, Handbook of Noise Control (McGraw-Hill, New York, 1957), Chap. 18.

2) A. London, "The determination of reverberant sound absorption coefficients from acoustic impedance measurements," J. Acoust. Soc. Am. 22, 263269 (1950).

3) U. Ingard and R. H. Bolt, "Absorption characteris- tics of acoustic material with perforated facing," J. Acoust. Soc. Am. 23, 533-540 (1951).

4) M. Ohta and T. Koizumi, "General statistical treatment of the response of a nonlinear rectifying device to a stationary random input," IEEE Trans. Inf. Theory IT-14, 595-598 (1968).

5) E. Lloyd, Handbook of Applicable Mathematics, Vol. II, Probability (John Wiley \& Sons, New York, 1980), p. 250.

6) E. Lloyd, Handbook of Applicable Mathematics, Vol. II, Probability (John Wiley \& Sons, New York, 1980), p. 252. 